\title{
Introduction to the Special Section on State and Parameter Estimation Methods for Sensorless Drives
}

Survey Article

\author{
Murat Barut ${ }^{1}$, Marko Hinkkanen², Teresa Orlowska-Kowalska³ \\ ${ }^{1}$ Department of Electrical and Electronics Engineering, Nigde Omer Halisdemir University, Nigde, Turkey \\ ${ }^{2}$ Department of Electrical Engineering and Automation, Aalto University, Aalto, Finland \\ ${ }^{3}$ Department of Electrical Machines, Drives and Measurements, Wroclaw University of Science and Technology, Wroclaw, Poland
}

Received August 09, 2018; Accepted October 05, 2018

\begin{abstract}
This short article constitutes an introductory part of the Special Section (SS) on State and Parameter Estimation Methods in Sensorless Drives. In the current issue of the journal, the first part of this section is published. Accepted articles are focussed mainly on estimation of the state variables and parameters for vector-controlled induction motor (IM) drives, using different concepts, such as different types of Kalman filters (KFs) and model reference adaptive systems (MRASs). The KF was also proposed for brushless DC motor (BLDC). Also, neural networks (NNs) have been proposed for mechanical state variables' estimation of the drive system with elastic couplings.

Keywords: sensorless control • extended Kalman filter • unscented Kalman filter • MRAS estimators • neural networks
\end{abstract}

Currently, speed or position sensorless drive has become crucial in industries in order to increase mechanical robustness and reliability of overall system and decrease its cost, size, and hardware complexity. However, the performance of sensorless control of electrical motors directly depends on the performance of state/parameter estimations, which is still problematic due to uncertainties in mathematical models, errors in measurements, variations in model parameters, and losing stability or observability around zero-speed range. Therefore, a great effort is needed in the literature to enhance the estimation performances of the states/parameters. This effort can be classified as deterministic- and stochastic-based estimator or observer designs using two-phase electrical motor model in addition to non-model-based high-frequency signal injection techniques.

The purpose of this special section (SS) was to reveal the recent findings to solve the problems associated with the sensorless estimation methods and thus high-performance and efficient sensorless electrical drives. The topics are focussed on:

- deterministic model-based methods such as model reference adaptive system (MRAS), adaptive full-order observers (AFOs), extended Leunberger observers (ELOs), and sliding mode observers (SMOs);

- stochastic model-based methods like extended Kalman filters (EKFs);

- deterministic/stochastic-based estimators or observers using artificial intelligence-based techniques;

- non-model-based methods; and

- other linear/non-linear design techniques.

In this issue of Power Electronics and Drives, we present the first part of this SS. Several papers of this SS are dedicated to state- and parameter-estimation problems in AC motor sensorless drives using different kinds of KFs. 
The first one by Zerdali and Barut proposes a novel EKF applied for estimation of electromagnetic and mechanical state variables and parameters in the induction motor (IM) drive. The proposed EKF simultaneously estimates the stator currents and rotor fluxes in stationary reference frames, rotor mechanical speed, load torque including the viscous friction term, and a reciprocal of total inertia of the drive system based on measured stator phase currents and voltages. The inertia estimation is done since it varies with the load coupled to the shaft and affects the performance of speed estimation, especially when the rotor speed changes. The EKF algorithm is supplemented with suitable observability analysis. Thus, in this article, the estimations of all mechanical state and parameters besides flux estimation required for high-performance control methods are performed together, and this solution is firstly introduced for high-performance speed-sensorless control of IMs. The performance of the proposed estimator is tested by simulation and real-time experiments under challenging variations in load torque and velocity references. Considering the obtained real-time results, the quite satisfactory estimation performance has been achieved in both transient and steady states, and the proposed method can operate between zero-speed and nominal-speed regions in which most of methods described in the literature fail. However, the proposed algorithm is still sensitive to the motor resistances and magnetising inductance (in a field weakening region); therefore, it requires the changing parameters' knowledge.

The KF is also used in the article by Horváth and Kuslits, describing the speed sensorless direct rotor fieldoriented control (DRFOC) system for the IM drive. The authors analyse and compare two solutions for estimation of IM electromagnetic state variables and load torque, namely, the conventional EKF and unscented Kalman filter (UKF). Both estimators are based on non-linear mathematical model of IM of the seventh order, which includes the non-linear static friction in the equation of motion. An important aspect is that one of the state variables is the load torque, and hence, it is also estimated by the estimator. Using this feature, the applied estimator-based speed sensorless control algorithm may be operated adequately, besides varying load torques. Dynamic performance of EKF and UKF are tested and compared by simulation and experimental tests. Results show that these two variants have comparable performance in general, but the UKF-based control provides better performance if a stochastically varying load disturbance is present.

An application of EKF is also experimented with by Chojowski, in the case of position and speed estimation of a BLDC motor. In this article, the BLDC motor state equations are developed and then expressed in a discretised form. EKF has been designed to observe specific states from the state vector, needed for the sensorless rotor position control and to determine the speed, which may be useful to use as a feedback for the controller. A test was carried out to determine the noise covariance matrices in a simulation manner. The process and measurement covariance matrices were selected using two methods: 1 - simulation way (time consuming), focussed on obtaining the smallest error value in the steady state and 2 - selecting the matrices in a way proposed in the article, based on a variance of the measurement noise. The second method is simpler and guarantees good estimation of the BLDC motor speed and position.

Next three articles accepted for this SS consider different MRAS techniques applied for IM drives with vector control.

The paper by Dybkowski proposes the novel concept of the universal speed and flux estimator associated with additional estimators of IM parameters, working sequentially. The proposed solution is based on the MRAS type flux and speed estimator, which uses the IM itself as a reference model and the stator current error combined with the estimated rotor flux for the speed adaptation mechanism (MRAS ${ }^{\mathrm{CC}}$ ). IM parameters are estimated using systems based only on the simple simulators and adaptive systems (voltage model and current model). The MRAS ${ }^{\mathrm{cC}}$ speed estimator combined with sequentially operating parameters' estimators constitutes the so-called universal estimator (UE). The proposed system was tested in the sensorless IM drive with Direct Field-Oriented Control (DFOC) algorithm in simulation and experimental tests. These tests show that magnetising reactance and the stator resistance estimator should be used simultaneously with the speed and flux estimators. Other parameter estimators constitute additional systems, which can be used for drive working in the heavy conditions. The main problem, which was marked in the paper, is connected with fast dynamical states. During this condition, the additional estimators provide the parameter values with relatively big errors and should be switched off during such operation states.

In the paper by Zorgani, Jouili, Koubaa and Boussak, the MRAS scheme is used to estimate simultaneously the speed and the rotor resistance in a sensorless indirect stator-flux-oriented control (ISFOC) IM drive at very low frequencies. The error between the reference and the adjustable models, which is developed in the stationary stator reference frame, is used to drive a suitable adaptation mechanism that generates the estimate speed and the rotor resistance from the stator voltage and the machine current measurement. The stator flux components 
in the stationary reference frame are estimated through a pure integration of the back Electro-Motive Force (EMF) of the machine. This method, however, has an error accumulation problem, especially when integrating at low speed. To overcome this problem, a strategy based on the stator flux synthesis through programmable cascaded low-pass filters (PCLPF) was proposed. The stability analysis method of the MRAS estimator is studied through the Lyapunov stability theory, in order to show the robustness of the proposed MRAS scheme to rotor resistance variations. The experimental results confirm the good dynamic performances of the developed estimator for the sensorless ISFOC IM drive in different operation conditions.

The paper by Korzonek and Orlowska-Kowalska considers the stability problems of different stator current error-based estimators of the IM speed, especially in the regenerating operation mode. The stability of the adaptive full-order observer (AFO) and two MRASs based on a stator current error (MRAS ${ }^{\mathrm{CC}}$ and MRAS ${ }^{\mathrm{CV}}$ ) is analysed, and the stability borders for these speed estimators are determined and compared based on the eigenvalues placement of the linearised state matrices of these speed estimators. It is shown that MRAS ${ }^{c V}$ speed estimator is stable in the whole operation range, including the regenerating mode without any modifications. The stability enhancement method for AFO and MRAS ${ }^{c C}$ estimators is described, and the solution for their stability improvement is proposed. Torque-speed characteristics of the analysed MRAS-type estimators in a wide range of drive speed and load torque changes are given, as well as the behaviour of estimators during transients is compared. The theoretical analysis and simulation test results are validated by experimental tests.

However, the MRAS ${ }^{c V}$ estimator is stable in the whole speed-torque range; experimental studies show that an important drawback of this speed estimator is the appearance of fixed angular velocity errors as the load torque increases. This disadvantage of MRAS ${ }^{\mathrm{CV}}$ is connected with a significant impact of motor parameter mismatch on the estimation quality of this estimator. The above-mentioned properties mean that, under real conditions, when motor parameters change due to variable operating points, the AFO and MRAS ${ }^{\mathrm{cC}}$ with proper method of stability improvement can behave better than the MRAS ${ }^{\mathrm{CV}}$ estimator.

The last paper in the first part of SS is focussed on the application of neural networks (NNs) for the state vector estimation of electrical drive with complex mechanical part. This paper presents results of research of the off-line trained, feedforward NN-based state estimator. The investigated system is the mechanical part of electrical drive characterised by elastic coupling with working machine, modelled as a dual-mass system. Mechanical resonance frequency of the system has been adopted at the level of $9.3 \mathrm{~Hz}$ to $10.3 \mathrm{~Hz}$. The aim of the research was to find a set of neural network structures giving useful and repeatable results of the mechanical state variable estimation. The authors give the recommendation for the NN structures and sizes considering the estimated mechanical state variable. Further work is oriented towards determining the upper limit of the resonance frequency of the mechanical drive with elastic couplings, for which static, neural models allow for state estimation.

The guest editors would like to thank both the authors for sending contributions and the reviewers for their expertise and time. 